\title{
Laparoscopic fistula excision and omentoplasty for high rectovaginal fistulas: a prospective study of 40 patients
}

\author{
Stephan J. van der Hagen - Peter B. Soeters • \\ Cor G. Baeten - Wim G. van Gemert
}

Accepted: 10 June 2011 / Published online: 24 June 2011

(C) The Author(s) 2011. This article is published with open access at Springerlink.com

\begin{abstract}
Aim The aim of this study is to prospectively evaluate 40 patients with a high rectovaginal fistula treated by a laparoscopic fistula division and closure, followed by an omentoplasty.

Patients and methods Forty patients with a rectovaginal fistula, between the middle third of the rectum and the posterior vaginal fornix, resulting from different causes (IBD, iatrogenic and birth trauma) were treated by a laparoscopic excision of the fistula and insertion of an omentoplasty in the rectovaginal septum. The patients completed the gastrointestinal quality of life index questionnaire (GIQLI) and the Cleveland Clinic incontinence score (CCIS). All tests were performed at regular intervals after treatment.

Results In 38 (95\%) patients with a median age of 53 years (range 33-72), the surgical procedure was feasible. In two patients, the fistula was closed without an omentoplasty, and a diverting stoma was performed. The median followup was 28 months (range 10-35). Two patients (5\%) developed a recurrent fistula. In one patient, the interposed omentum became necrotic and was successfully treated laparoscopically. In another patient, an abscess developed, which needed drainage procedures. The mean CCIS was 9 (range 7-10) before treatment and 10 (range 7-13) after treatment ( $p=0.5$ Wilcoxon). The median GIQLI score was 85 (range 34-129) before treatment and 120 (range 75-142) after treatment ( $p=0.0001$, Wilcoxon).
\end{abstract}

S. J. van der Hagen $(\bowtie) \cdot$ P. B. Soeters • C. G. Baeten •

W. G. van Gemert

Department of Surgery, Refaja Hospital Stadskanaal,

University Hospital of Maastricht,

Boerhaavestraat 1, 9501 HE Stadskanaal,

Maastricht, Netherlands

e-mail: manonstephan@kpnplanet.nl
Conclusions Laparoscopic fistula excision combined with omentoplasty is a good treatment modality with a high healing rate for high rectovaginal fistulas and an acceptable complication rate.

Keywords Omentoplasty - Omentum · Laparoscopic fistula excision $\cdot$ Rectovaginal fistulas $\cdot$ Rectovaginal septum

\section{Introduction}

Rectovaginal fistulas significantly reduce the quality of life of affected women and represent a challenge for surgeons. The vaginal passage of gas and stool can cause physical symptoms due to inflammation and irritation. Patients may also suffer from significant psychosocial and sexual dysfunction [1].

The treatment of rectovaginal fistulas depends on size, location, cause, continence, and overall health status of the patient. The transanal endorectal advancement flap is the most popular technique to repair simple low rectovaginal fistulas by colorectal surgeons, while the gynecologists prefer the transvaginal approach. The transabdominal approach is the standard surgical approach for high fistulas with large defects $[2,3]$. This technique includes fistula division and closure with or without bowel resection and interposition of local flaps, such as muscle and musculocutaneous flaps. In most techniques, diversion colostomy is preferred to safeguard the procedure $[2,3]$.

In the present study, no diversion colostomy was deemed necessary if the omentum is interposed between the properly sutured defects remaining after fistula division and excision. The laparoscopic repair of rectovaginal fistulas has all the advantages of minimal access 
surgery. It is associated with minimal wound complications, less postoperative pain, and early discharge of the patient. The technique can replace the transanal or transvaginal approach if the rectovaginal septum is opened as distal as the pelvic floor. In this study, we investigated the results of a laparoscopic transabdominal approach in patients with high rectovaginal fistulas by excision and closure of the fistulous tract followed by an omentoplasty.

\section{Patients and methods}

Between 2006 and 2009, patient characteristics like age, fistula cause, and previous surgical attempts were recorded of all consecutive patients with a rectovaginal fistula. The patients underwent physical examination, transvaginal ultrasound, and proctosigmoidoscopy as a standard procedure. Because high rectovaginal fistulas can be easily missed by both physical examination and endoscopy, methylene blue enemas with a vaginal tampon in place were used for the confirmation of diagnosis. A fistula between the middle third of the rectum and the posterior vaginal fornix was defined as a high fistula.

Only patients with a confirmed high fistula were recruited for this study. The study protocol was approved by the ethical committee. Written informed consent was obtained before entering the study. Before treatment and at 12 months after treatment, the patients filled out the gastrointestinal quality of life index questionnaire and the Cleveland Clinic incontinence score. Patients younger than 18, patients with malignancy, patients that had received radiation therapy, or patients who were HIV-infected were excluded.

\section{Procedure}

After the induction of general anaesthesia, 2,200 mg amoxicillin/clavulanic acid was given intravenously. Cleansing of the vaginal lumen with an antiseptic solution (povidone-iodine) was done. A Foley catheter was inserted into the urinary bladder. The procedure was performed with the patient in a semi-lithotomy position, with the legs in a downwards position. After reaching the pneumoperitoneum, the patient was placed in a Trendelenburg position. Four trocars were placed. The ileum loops were gently moved out of the pelvic cavity, and the sigmoid loop is withdrawn in a left cranial position.

Possible adhesions between the rectum and the bladder peritoneum were dissected. A peritoneal incision was made over the sacral promontory and extended caudally along the rectum over the deepest part of the pouch of Douglas. Denonvilliers' fascia was incised. The (fibrotic) fistula was identified and was divided. After the fistulous tract was opened, the rectal and vaginal sides of the fistula were identified. The fistula tract was resected, and the rectovaginal septum broadly opened down to the pelvic floor.

The opening of the fistula on the rectal side was closed in two layers with 2-0 Vicryl by intracorporeal suturing. The vaginal side was closed with $2-0$ Vicryl in a single continuous layer.

After placing the patient in the anti-Trendelenburg position, the omentum was dissected free from the transverse colon. The right or left gastroepiploic artery is dissected, and the omentum was mobilized by cutting the gastroepiploic arcade at one side. Subsequently, the omentum is brought down to the pelvis either by tunneling it through the mesocolon or positioning it in the left or right paracolic gutters. Finally, the omentum was placed between the repaired rectum and the repaired vagina and sutured to the pelvic floor. The abdominal cavity was rinsed with 21 of $\mathrm{NaCl}$. A drain was placed in the pelvis through the right flank and removed within 2 days when discharge was negligible.

\section{Intention to treat}

In this study, the results of all patients that were included to undergo this procedure were analyzed. If an omentoplasty was not feasible, the fistula was closed and a diverting stoma was performed.

\section{Examination}

Patients were followed at regular intervals of 2 months at the outpatient clinic and were re-examined for the purpose of this study. The fistula was considered to be healed if there were no passage of gas and stool and no physical symptoms due to inflammation and irritation. All of the patients received after 6 months a methylene blue rectal enema and a vaginal tampon to confirm that the fistula was closed. Continence disorders were examined by the Cleveland Clinic incontinence score before treatment and at 12 months after treatment. Before treatment and 12 months after treatment, the patients filled in the gastrointestinal quality of life index questionnaire. Hospital stay, operation time, and complications were recorded.

\section{Statistical analysis}

Comparisons were made between the outcome obtained before treatment and at 12 months after treatment. Statistical analysis included two-tailed $t$ tests and two-way analysis for the observed changes in the faecal incontinence and quality of life scales. A probability of 0.05 was considered significant. 
Table 1 Patients' characteristics and outcome

\begin{tabular}{lcccc}
\hline & IBD, $N=3(8 \%)$ & Birth trauma, $N=19(47 \%)$ & Postsurgery, $N=18(45 \%)$ & $N=40$ \\
\hline$>1$ previous surgical attempt in history & 3 & 10 & 8 & 2 \\
Failure of omentoplasty & 0 & 0 & 1 & $2(5 \%)$ \\
Recurrent fistula & 1 & 0 & $4(3-7)$ & $4(5 \%)$ \\
Hospital stay (days) & $4(3-5)$ & $4(2-6)$ & $145(128-173)$ & $128(118-173)$ \\
Operation time (min) & $127(120-135)$ & $127(118-140)$ & & \\
\hline
\end{tabular}

\section{Results}

Forty consecutive patients with a median age of 53 years (range 33-72) with a rectovaginal fistula were included. The patients' characteristics and outcomes are described in Table 1.

In 38 (95\%) patients, the laparoscopic omentoplasty was feasible. The median hospital stay was 4 days (range 2-7). The median operation time amounted to 133 min (range 118-173). The median follow-up was 28 months (range $10-35)$.

There was no difference in incontinence disorders before and after treatment. The mean CCIS was 9 (range 7-10) before treatment and 10 (range 7-13) after treatment $(p=0.5$, Wilcoxon).

The median GIQLI score was 85 (range 34-129) before treatment and 120 (range75-142) after treatment ( $p=0.0001$, Wilcoxon). The domain scores are listed in Table 2.

\section{Complications}

In two patients, the fistula was closed without an omentoplasty because of an unsuitable omentum. In these patients, a diverting stoma was performed. Two patients (5\%) developed a recurrent fistula during follow-up. The patient with a recurrent fistula in the postsurgery group did

Table 2 GIQLI score and CCIS

\begin{tabular}{lccc}
\hline & \multicolumn{2}{l}{$\begin{array}{l}\text { Total gastrointestinal quality of life index and } \\
\text { domain scores and CCIS }\end{array}$} \\
\cline { 2 - 4 } & Preoperative & After treatment & $P$ value \\
\hline Total GIQLI score & $85(34-129)$ & $120(75-142)$ & $<0.00001$ \\
Symptoms & $44(21-670)$ & $62(41-74)$ & $<0.00001$ \\
Physical function & $13(3-26)$ & $23(7-28)$ & $<0.00001$ \\
Social function & $10.5(3-15)$ & $14(8-17)$ & $<0.0001$ \\
Emotional function & $11(1-19)$ & $17(5-21)$ & $<0.00001$ \\
Subjective treatment & $3(0-4)$ & $4(1-4)$ & $<0.00015$ \\
$\quad \begin{array}{l}\text { assessment } \\
\text { CCIS }\end{array}$ & $9($ range $7-10)$ & $10($ range $7-13)$ & 0.5 \\
\hline
\end{tabular}

not want a new surgical intervention because of minor complaints of the fistula. The patient with the recurrent fistula and Crohn's disease underwent a diverting stoma.

In one patient, a necrotic omentum developed. This was successfully treated by a laparoscopic necrotectomy. In another patient, an abscess developed, which needed drainage procedures. Minor complications included gastric retention and urinary infection (Table 3 ).

\section{Discussion}

Rectovaginal fistulas can develop from several conditions, including obstetric trauma, inflammatory bowel disease (IBD), carcinoma, radiation, diverticulitis, and infectious processes, and as a result of postsurgical procedures. The most common cause of rectovaginal fistulas is obstetric trauma because of prolonged labor. Predisposing factors include forceps delivery, midline episiotomy, and third- or fourth-degree perineal lacerations [4]. The second most common cause of rectovaginal fistulas is inflammatory bowel disease, specifically Crohn's disease. Rectovaginal fistulas have been reported to occur in $9-10 \%$ of patients. Radcliffe et al. reported an incidence of $9.8 \%$ of rectovaginal fistulas in women with Crohn's disease [5]. Malignant processes, including cancer of the rectum, cervix, uterus, or vagina, can also contribute to the development of a rectovaginal fistula. In addition, the fistulas can develop as complications of radiation therapy and postsurgical complications including low anterior resection with stapled anastomosis, hysterectomy, rectocele repair, and restorative proctocolectomy with ileal pouch anastomosis.

In the present study, a relatively high number of patients [18 (45\%)] were treated for a rectovaginal fistula because of postsurgical complications after hysterectomy

Table 3 Minor complications

\begin{tabular}{ll}
\hline Stomach distension & 5 \\
Trocar wound infection & 1 \\
Urinary tract infection & 3 \\
\hline
\end{tabular}


and/or rectocele repair. In ten of these patients, not only the fistula was excised but also the mesh had to be removed, which required a prolonged operation time in this special group. Rectovaginal fistulas resulting from radiation therapy are treated in our clinic by bowel resection and colostomy.

The successful management of rectovaginal fistulas depends on a variety of factors including etiology, size, and location of the fistula. Especially, the management of rectovaginal fistulas in patients with Crohn's disease is still extremely challenging and the recurrence rate varies between $50 \%$ and $100 \%[6,7]$. The recurrence rate of traumatic and obstetric rectovaginal fistulas varies between $11 \%$ and $25 \%[6,8]$.

Various therapies have been proposed, such as advancement flap plasties or bioprosthetic plugs, applied transvaginally or transanally especially for the treatment of low rectovaginal fistulas [9-14]. In patients with high rectovaginal fistulas, especially in Crohn's disease, the transabdominal approach is the most frequently published. Bowel resections, Gracilis transposition, proctectomy, and diversion colostomy are commonly performed when cure is not to be expected $[2,3,14,15]$.

Rectovaginal fistulas in IBD are frequently treated by infliximab without surgery. The closure rate is about $30-50 \%$ $[1,16,17]$. Another study suggested the effectiveness of staged definitive surgery combined with infliximab in the treatment of patients with Crohn's disease [18]. In the present study, only three $(8 \%)$ patients with IBD were included: two patients with Crohn's disease and one with ulcerative colitis. One patient had been treated previously with infliximab, but all three patients with IBD were on maintenance therapy with azathioprine and one with corticosteroids. None of them had active rectal disease at the time of treatment by laparoscopic omentoplasty.

Several studies have reported cases of rectovaginal fistula repair by laparoscopy [19-21]. Pelosi et al. performed laparoscopic upper rectovaginal mobilization to facilitate the transvaginal repair of recurrent rectovaginal fistula [22]. Schwenk et al. reported a case of a rectovaginal fistula for which they had performed a laparoscopic resection of the sigmoid colon which included the fistulous tract, after which an intracorporeal colorectal anastomosis was performed together with an omentoplasty [23].

The effectiveness of intra-abdominal use of an omentoplasty is described in several studies in patients especially with hydatic cysts to overcome surgical site infections and fistulas [24-27], but no series of patients with rectovaginal fistulas, treated by complete laparoscopic repair or treated by omentoplasty, have been published in the literature.

Our study shows that laparoscopic fistula excision and omentoplasty for high rectovaginal fistulas can be performed safely and with a short hospital stay, a low recurrence rate, and without requiring a diverting colostomy.

Open Access This article is distributed under the terms of the Creative Commons Attribution Noncommercial License which permits any noncommercial use, distribution, and reproduction in any medium, provided the original author(s) and source are credited.

\section{References}

1. Sands BE et al (2004) Long-term treatment of rectovaginal fistulas in Crohn's disease: response to infliximab in the ACCENT II Study. Clin Gastroenterol Hepatol 2(10):912-920

2. Athanasiadis $\mathrm{S}$ et al (2007) Recovery rates and functional results after repair for rectovaginal fistula in Crohn's disease: a comparison of different techniques. Int $\mathrm{J}$ Colorectal Dis 22 (9):1051-1060

3. Mueller MH et al (2007) Risk of fecal diversion in complicated perianal Crohn's disease. J Gastrointest Surg 11(4):529-537

4. Tebeu PM et al (2009) Risk factors for obstetric fistula in the Far North Province of Cameroon. Int J Gynaecol Obstet 107(1):12-15

5. Radcliffe AG et al (1988) Anovaginal and rectovaginal fistulas in Crohn's disease. Dis Colon Rectum 31(2):94-99

6. Pinto RA et al (2010) Are there predictors of outcome following rectovaginal fistula repair? Dis Colon Rectum 53 (9): $1240-1247$

7. Ruffolo C et al (2009) A systematic review on advancement flaps for rectovaginal fistula in Crohn's disease: transrectal versus transvaginal approach. Colorectal Dis 12(12):1183-1191

8. Baig MK et al (2000) Simple rectovaginal fistulas. Int J Colorectal Dis 15(5-6):323-327

9. de Parades V et al (2011) Endorectal advancement flap with muscular plication: a modified technique for rectovaginal fistula repair. Colorectal Dis. doi:10.1111/j.1463-1318.2010.02338.x

10. Ellis CN (2008) Outcomes after repair of rectovaginal fistulas using bioprosthetics. Dis Colon Rectum 51(7):1084-1088

11. Ellis CN, Clark S (2007) Effect of tobacco smoking on advancement flap repair of complex anal fistulas. Dis Colon Rectum 50(4):459-463

12. Halverson AL et al (2001) Repair of recurrent rectovaginal fistulas. Surgery 130(4):753-757, discussion 757-758

13. Khanduja KS et al (1994) Delayed repair of obstetric injuries of the anorectum and vagina. A stratified surgical approach. Dis Colon Rectum 37(4):344-349

14. Loffler $\mathrm{T}$ et al (2009) Long-term success rate after surgical treatment of anorectal and rectovaginal fistulas in Crohn's disease. Int J Colorectal Dis 24(5):521-526

15. Lefevre JH et al (2009) Operative results and quality of life after gracilis muscle transposition for recurrent rectovaginal fistula. Dis Colon Rectum 52(7):1290-1295

16. Topstad DR et al (2003) Combined seton placement, infliximab infusion, and maintenance immunosuppressives improve healing rate in fistulizing anorectal Crohn's disease: a single center experience. Dis Colon Rectum 46(5):577-583

17. Ardizzone S et al (2004) Perianal fistulae following infliximab treatment: clinical and endosonographic outcome. Inflamm Bowel Dis 10(2):91-96

18. van der Hagen SJ et al (2005) Anti-TNF-alpha (infliximab) used as induction treatment in case of active proctitis in a multistep strategy followed by definitive surgery of complex anal fistulas in 
Crohn's disease: a preliminary report. Dis Colon Rectum 48 (4):758-767

19. Nezhat $\mathrm{CH}$ et al (1998) Laparoscopic treatment of type IV rectovaginal fistula. J Am Assoc Gynecol Laparosc 5(3):297-299

20. Kumaran SS et al (2005) Laparoscopic repair of high rectovaginal fistula: is it technically feasible? BMC Surg 5:20

21. Palanivelu $C$ et al (2007) Laparoscopic management of iatrogenic high rectovaginal fistulas (type VI). Singap Med J 48(3):e96-e98

22. Pelosi MA 3rd, Pelosi MA (1997) Transvaginal repair of recurrent rectovaginal fistula with laparoscopic-assisted rectovaginal mobilization. J Laparoendosc Adv Surg Tech A 7(6):379-383

23. Schwenk W et al (1997) Laparoscopic resection of high rectovaginal fistula with intracorporeal colorectal anastomosis and omentoplasty. Surg Endosc 11(2):147-149
24. Bickel A et al (2001) The laparoscopic approach to abdominal hydatid cysts: a prospective nonselective study using the isolated hypobaric technique. Arch Surg 136(7):789-795

25. Dziri C et al (1999) Omentoplasty in the prevention of deep abdominal complications after surgery for hydatid disease of the liver: a multicenter, prospective, randomized trial. French Associations for Surgical Research. J Am Coll Surg 188 (3):281-289

26. Fujiwara K et al (2003) Effect of simple omentoplasty and omentopexy in the prevention of complications after pelvic lymphadenectomy. Int J Gynecol Cancer 13(1):61-66

27. Muftuoglu MA, Koksal N, Topaloglu U (2005) The role of omentoplasty in the surgical management of remnant cavity in hepatic hydatid cyst. HPB (Oxford) 7(3):231-234 